\title{
Fiber Bragg grating as an optical filter tuned by a magnetic field
}

\author{
J. L. Arce-Diego, R. López-Ruisánchez, and J. M. López-Higuera
}

Grupo de Ingeniería Fotónica, Departamento de Tecnología Electronica e Ingenería de Sistemas Automatíca, Universidad de Cantabria, Avenida Los Castros s/n., Santander 39005, Spain

\author{
M. A. Muriel \\ Departamento Tecnología Fotónica, Universidad Politécnica de Madrid, Madrid 28040, Spain
}

\author{
Received December 4, 1996
}

We describe a novel tunable optical filter for use in optical-frequency-domain multiplexed communication systems. The shift in the Bragg condition of a fiber Bragg grating as a result of magnetically induced circular birefringence is calculated with coupled-mode theory on the basis of circular states of polarization, and the values obtained for silica and terbium-doped optical fibers are compared. (c) 1997 Optical Society of America

Fiber Bragg gratings (FBG's) are currently used in sensing (strain and temperature, ${ }^{1}$ magnetic field ${ }^{2}$ ) and optical communication systems. In-fiber Bragggrating sensors have attracted considerable interest because of their intrinsic nature and wavelengthencoded operation. In this Letter we demonstrate the ability to implement tunable optical filters that use such devices. These new tunable optical filters are based on the Bragg-condition shift of a fiber-optic Bragg grating without linear birefringence ${ }^{3}$ perturbed by variable magnetic fields.

An electromagnetic field in anisotropic single-mode fibers (perturbed systems) can be represented as a linear combination of round-core fiber (unperturbedsystem) fields ${ }^{4}$ by

$$
\begin{aligned}
\mathbf{E} & =\left[A(z) \mathbf{e}_{1}+B(z) \mathbf{e}_{2}\right] \exp (j \omega t), \\
\mathbf{H} & =\left[A(z) \mathbf{h}_{1}+B(z) \mathbf{h}_{2}\right] \exp (j \omega t) .
\end{aligned}
$$

Here $\left\{\mathbf{e}_{1}, \mathbf{h}_{1}\right\}$ and $\left\{\mathbf{e}_{2}, \mathbf{h}_{2}\right\}$ denote two eigenfunctions in the unperturbed fiber and satisfy an orthonormalization relationship to each other. The coefficients $A(z)$ and $B(z)$ are the field amplitudes, which depend on the fiber-axis coordinate $z$.

The dielectric-constant tensor $\Delta \epsilon$ represents a deviation from the unperturbed system. We derived the fields in the perturbed system by substituting Eqs. (1) into Maxwell's equations and the material equations and regarding $\Delta \epsilon$ as a perturbation.

The behavior of $A(z)$ and $B(z)$ is represented by the simple coupled-mode equations

$$
\frac{\mathrm{d}}{\mathrm{d} z}\left(\frac{A}{B}\right)=-j\left[\begin{array}{ll}
\kappa_{11} & \kappa_{12} \\
\kappa_{21} & \kappa_{22}
\end{array}\right]\left(\begin{array}{l}
A \\
B
\end{array}\right)
$$

with

$$
\begin{gathered}
\kappa_{i i}=\beta_{0}+\omega \epsilon_{0} \int \mathbf{e}_{i}^{*} \Delta \epsilon \mathbf{e}_{i} \mathrm{~d} S, \quad i=1,2, \\
\kappa_{i j}=\kappa_{j i}{ }^{*}=\omega \epsilon_{0} \int \mathbf{e}_{i}^{*} \Delta \epsilon \mathbf{e}_{j} \mathrm{~d} S, \quad i \neq j,
\end{gathered}
$$

where the asterisks indicate complex conjugation, $\beta_{0}$ is the propagation constant in the unperturbed sys- tem, $\epsilon_{0}$ is the electric permittivity in vacuum, $\omega$ is the angular frequency, and $S$ is the cross section of the fiber.

Coupled-mode theory ${ }^{5}$ is a powerful tool for studying the change in light propagation caused by perturbations of geometry of material properties, and it is most suitable for describing the superposition of different perturbations in single-mode fibers, given by their respective dielectric tensors, which will change the polarization properties of the fiber. For the correct application of coupled-mode theory, a natural frame has to be used to represent the basic modes of the nonperturbed fiber and the dielectric tensor of the perturbation. Tang showed ${ }^{6}$ that there is a natural frame in a nonperturbed (isotropic) waveguide that follows an arbitrarily curved line in space, for which the solutions of the wave equation become orthogonal.

When a magnetic field $H_{z}$ in the direction of the light propagation, $z$, is applied to an optical fiber, the Faraday effect induces a circular-birefringence perturbation given by the dielectric tensor

$$
\Delta \epsilon=\left[\begin{array}{ccc}
0 & -\Delta \epsilon_{F} & 0 \\
\Delta \epsilon_{F} & 0 & 0 \\
0 & 0 & 0
\end{array}\right],
$$

with $\Delta \epsilon_{F}=2 j n_{0} V H_{z} / k$, where $V$ is the Verdet constant and $k=\omega / c$. The typical values for the Verdet constant in single-mode fibers can be approximately expressed as $V=0.14 \times 10^{-32} c^{2} / \lambda^{2} \mathrm{rad} /($ Gauss m).

The basis of the fundamental modes $\mathrm{e}_{1}$ and $\mathrm{e}_{2}$ is usually chosen to describe orthogonal linear polarization; this choice is arbitrarily adapted to the anisotropy involved. Since the Faraday effect induces circular birefringence, and since the eigenmodes of the rotation effects are circular, it is most convenient to express $\mathbf{E}$ on the basis of orthogonal left- and right-circular polarizations. The coupled-mode approach now assumes that the electric field $\mathbf{E}$ in the perturbed medium is given by a linear superposition of the nonperturbed modes:

$\mathbf{E}=C_{+} \exp \left[j\left(w t-\beta_{+} z\right)\right] \mathbf{R}+C_{-} \exp \left[j\left(w t-\beta_{-} z\right)\right] \mathbf{L}$. 
Then, assuming single-mode fibers and two orthogonal circular polarizations, $R$ and $L$, we can write the coupled equations for the amplitudes $C_{+}$and $C_{-}$of two such modes as

$$
\frac{\mathrm{d}}{\mathrm{d} z}\left(\begin{array}{l}
C_{+} \\
C_{-}
\end{array}\right)=j\left[\begin{array}{ll}
\kappa_{++} & \kappa_{+-} \\
\kappa_{-+} & \kappa_{--}
\end{array}\right]\left(\begin{array}{l}
C_{+} \\
C_{-}
\end{array}\right)
$$

where $\kappa_{i j}(i, j=+,-)$ are the coupling coefficients.

From Eqs. (3) and (4), on a circular basis, one finds the coupling coefficients for the Faraday effects for light propagating in opposite directions, $+z$ and $-z$ :

$$
\overrightarrow{K_{F}}=\left[\begin{array}{cc}
V H_{z} & 0 \\
0 & -V H_{z}
\end{array}\right], \quad \overleftarrow{K_{F}}=\left[\begin{array}{cc}
V H_{z} & 0 \\
0 & -V H_{z}
\end{array}\right]
$$

where $H_{z}$ is the magnetic-field component in the direction of the $z$ axis. For both directions of the propagation the two circular polarizations are not coupled, but they will propagate with different speeds.

The refractive indices for the $R$ mode $n^{+}$and the $L$ mode $n^{-}$, for small perturbation, are given by ${ }^{7}$

$$
n_{f}^{+}=n+\frac{\gamma H_{z}}{2 n}, \quad n_{f}^{-}=n-\frac{\gamma H_{z}}{2 n},
$$

where $\gamma$ is the magnetogyration coefficient of the medium.

The specific rotatory power in a medium with circular birefringence (the rotation per unit length of the linearly polarized electric field that propagates in the medium with optical activity) is given by

$$
\rho=\frac{\pi}{\lambda}\left(n^{+}-n^{-}\right),
$$

and, as the specific rotation for the Faraday effect is often written as $\rho=V H_{z}$, the circular birefringence induced by the Faraday effect becomes

$$
n_{f}^{+}=n+\frac{V H_{z} \lambda}{2 \pi}, \quad n_{f}^{-}=n+\frac{V H_{z} \lambda}{2 \pi} ;
$$

then

$$
n_{f}^{+}-n_{f}^{-}=V H_{z} \lambda / \pi .
$$

When a magnetic field is applied to a FBG (a periodically layered medium induced in the core of the singlemode optical fiber), the circular birefringences $n_{1 f}^{+}, n_{1 f}^{-}$ and $n_{2 f}^{+}, n_{2 f}^{-}$are induced in layers 1 and 2 , respectively, of that FBG (Fig. 1).

Bragg reflection is a typical example of contradirectional coupling. ${ }^{7} \quad$ Therefore we can use the coupledmode theory to treat the optical properties of a shallow Bragg reflector perturbed by a magnetic field. The normal modes in the FBG with $H_{z}$ are plane waves $\exp (-j \mathbf{k} \cdot \mathbf{r})$ with circular states of polarization and wave numbers given by

$$
\begin{aligned}
& k^{+2}=\left(\frac{\omega}{c}\right)^{2} \frac{n_{1}^{+2}+n_{2}^{+2}}{2} \equiv\left(\bar{n}^{+} \frac{\omega}{c}\right)^{2}, \\
& k^{-2}=\left(\frac{\omega}{c}\right)^{2} \frac{n_{1}^{-2}+n_{2}^{-2}}{2} \equiv\left(\bar{n}^{-} \frac{\omega}{c}\right)^{2},
\end{aligned}
$$

where $\bar{n}^{+}$and $\bar{n}^{-}$are the geometric average refractive indices of the medium with the Faraday effect.
Since both perturbed and nonperturbed dielectric constants are scalar, mode coupling between $R$ and $L$ waves does not exist. Consequently, only coupling between waves of the same polarization state can take place (Fig. 2). This is possible only in the contradirectional coupling, because the phase-matching condition for codirectional coupling can never be satisfied; $\Delta \beta=\beta^{+}-\beta^{-}-m(2 \pi / \Lambda)=-m(2 \pi / \Lambda)=0$ for finite $\Lambda$. Maximum reflectivity occurs at the Bragg wavelength, when $\Delta \beta=0 . \quad \beta^{+}$and $\beta^{-}$are the propagation constants of the circular-polarization modes.

Thus, in the presence of magnetic fields applied to the FBG, the index is changed for the two circular polarizations, and consequently two Bragg conditions ${ }^{8}$ are observed (Fig. 3):

$$
\lambda_{B f}^{+}=2 n_{f}^{+} \Lambda, \quad \lambda_{B f}^{-}=2 n_{f}^{-} \Lambda .
$$

Based on this effect, it is possible to implement a new tunable optical filter. The tuning of the new tunable optical filter that is proposed is based on the change in index induced by a magnetic field applied to a FBG.

The range for tuning, when $\Lambda$ is given, is then dependent on parameters $V$ and $H_{z}$. Thus, it is possible for one to select the central wavelength of the proposed filter by modifying these parameters:

$$
\left|\lambda_{B f}^{+}-\lambda_{B f}^{-}\right|=2 \Lambda V H_{z} \lambda / \pi \text {. }
$$

In silica fibers with $\lambda=1.3 \mu \mathrm{m}$, the Verdet constant is $V \approx 8 \times 10^{-5} \mathrm{rad} /(\mathrm{G} \mathrm{m})$, so a FBG $(\Lambda \approx 1 \mu \mathrm{m})$

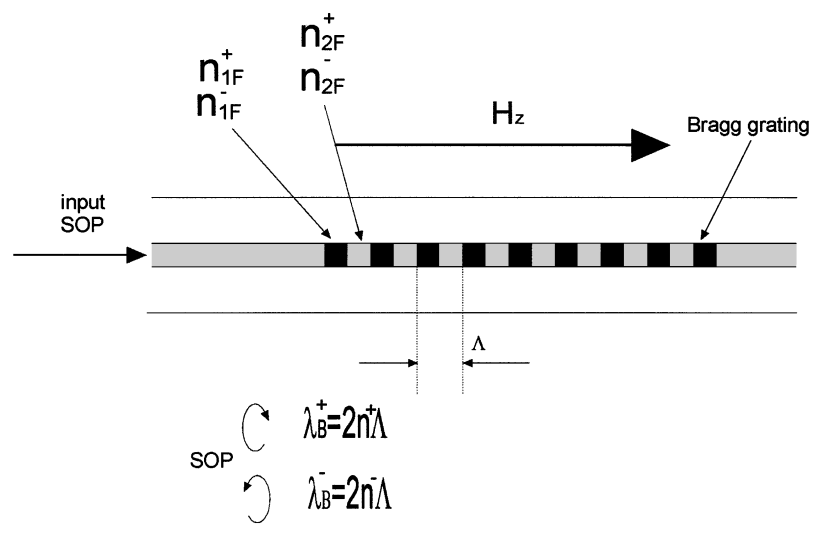

Fig. 1. Circular birefringence induced by the Faraday effect on each FBG layer. SOP, state of polarization.

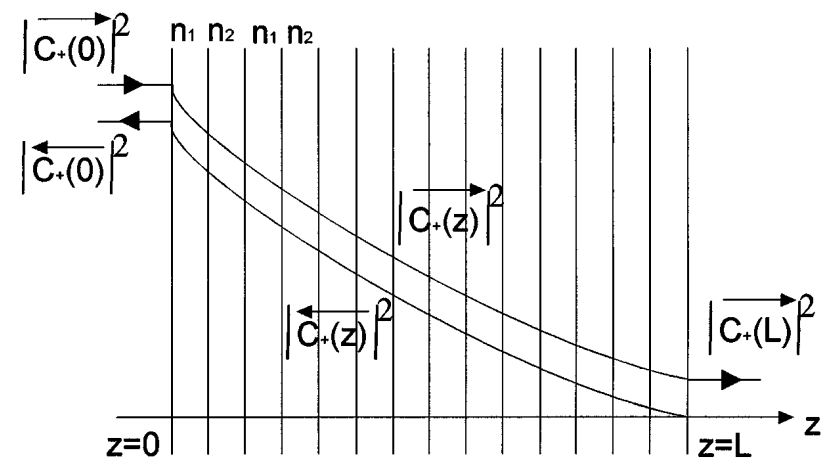

Fig. 2. Powers of the incident and reflected circularly polarized modes in a periodic layered medium when $\Delta \beta=0$. 


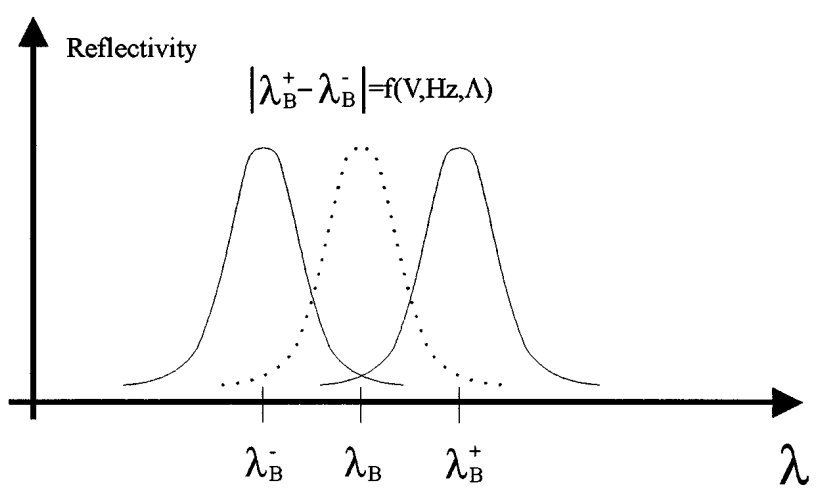

Fig. 3. Wavelength shift induced by the Faraday effect in the FBG.

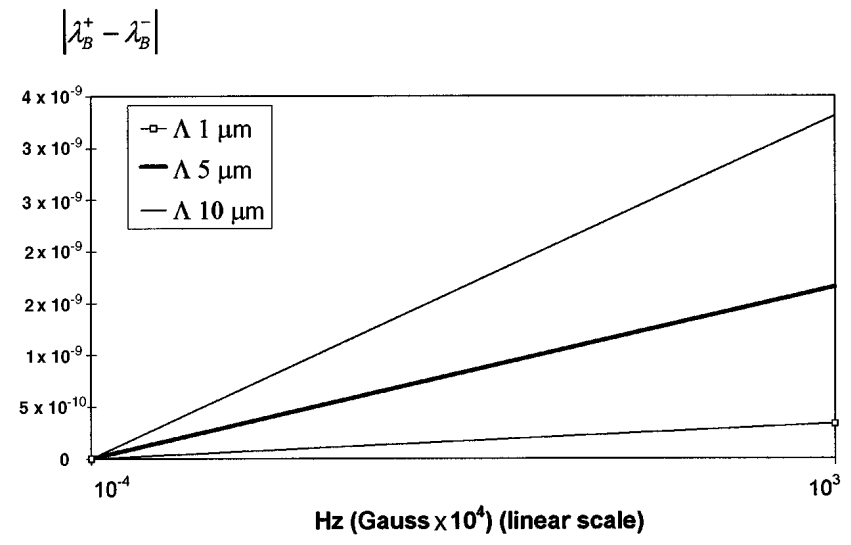

Fig. 4. Bragg condition shift versus magnetic field in a standard optical fiber.

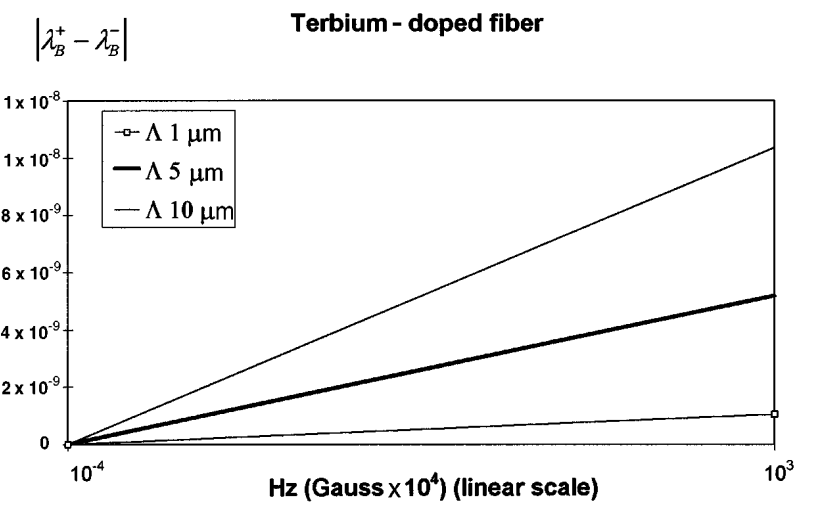

Fig. 5. Bragg condition shift versus magnetic field in a terbium-doped optical fiber.

needs high magnetic fields, $H_{z} \approx 750 \mathrm{G}$, for a Braggcondition shift of $10 \mathrm{MHz}(\sim 0.00005 \mathrm{~nm})$, and its dependence on $H_{z}$ is represented for different pitches in Fig. 4. One solution is to use a terbium-doped optical fiber with $V \approx 2.5 \times 10^{-4} \mathrm{rad} /(\mathrm{G} \mathrm{m})$; in this case, for the same FBG and Bragg-condition shift, $H_{z} \approx$ $240 \mathrm{G}$, at the same wavelength (Fig. 5). Another way of creating a larger Bragg-condition shift is to use a multilayer Bragg grating, whose pitches can be larger than FBG pitches, that is composed of paramagnetic

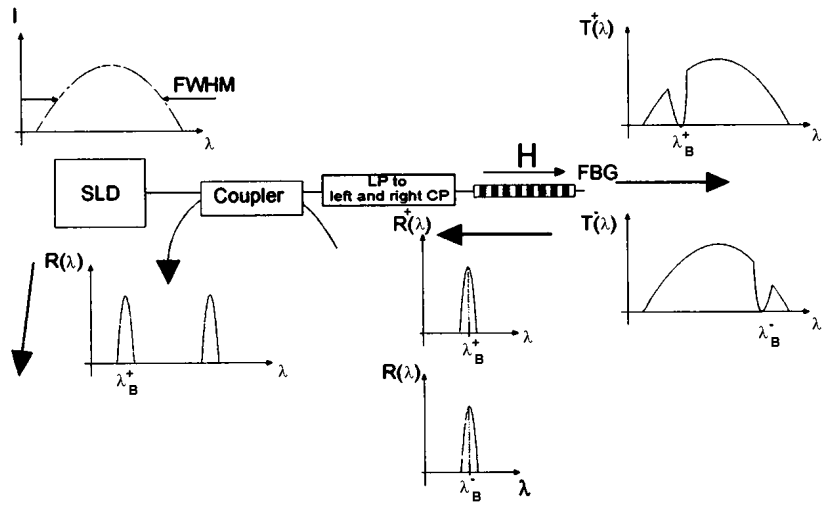

Fig. 6. Spectral response of the proposed optical filter. LP, left polarization; CP, circular polarization; SLD, superluminescent diode.

or ferromagnetic optical materials with a larger Verdet constant than silica fibers.

The spectral response of this device is shown in Fig. 6, in which the left-polarization-circularpolarization converter is an optical circuit that transforms the incident linearly polarized light into the right and left circularly polarized modes that interact with the FBG exposed to the magnetic field.

In conclusion, a new tunable optical filter based on Faraday effect on Bragg gratings, useful for wavelength fine tuning in optical circuits, for sensors, and for optical communication systems has been studied. Our results have shown that its implementation in FBG necessitates high magnetic fields. However, by use of optical materials with a high Verdet constant, its further development is possible.

This work was sponsored by the Spanish Ministry of Education and Science through Comisión Interministerial de Ciencia y Tecnología project TIC950631-C04-01.

\section{References}

1. V. W. Morey, G. Meltz, and W. H. Green, in Proceedings of Optical Fiber Sensors '89, Vol. 44 of Springer Proceedings in Physics, (Springer-Verlag, Berlin, 1989), p. 526.

2. A. D. Kersey and M. J. Marrone, Proc. SPIE 2360, 53 (1994).

3. T. Meyer, P.-A. Nicati, P. A. Robert, D. Varelas, H.-G. Limberger, and P. Salathé, Opt. Lett. 21, 1661 (1996).

4. J. Sakai and T. Kimura, IEEE J. Quantum Electron. 18, 59 (1982).

5. A. W. Snyder and J. D. Love, Optical Waveguide Theory (Chapman \& Hall, London, 1996).

6. C. Tang, IEEE Trans. Microwave Theory Technol. MTT18, 69 (1970).

7. A. Yariv and P. Yeh, Optical Waves in Crystals: Propagation and Control of Laser Radiation (Wiley-Interscience, New York, 1984).

8. J. L. Arce-Diego, J. M. López-Higuera, and M. A. Muriel, in Proceedings of the Laser and Electro-Optics Society (Institute of Electrical and Electronics Engineers, New York, 1996), p. 307. 